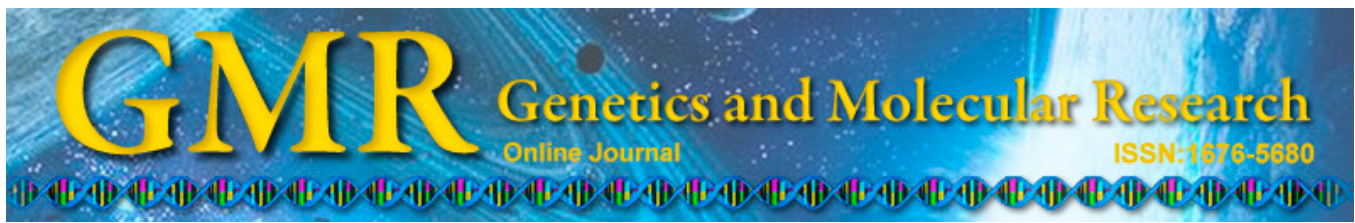

\title{
OsMYB511 encodes a MYB domain transcription activator early regulated by abiotic stress in rice
}

\author{
P. Huang, H. Chen, R. Mu, X. Yuan, H.S. Zhang and J. Huang \\ State Key Laboratory of Crop Genetics and Germplasm Enhancement, \\ Nanjing Agricultural University, Nanjing, China \\ Corresponding author: J. Huang \\ E-mail: huangji@njau.edu.cn
}

Genet. Mol. Res. 14 (3): 9506-9517 (2015)

Received November 24, 2014

Accepted April 6, 2015

Published August 14, 2015

DOI http://dx.doi.org/10.4238/2015.August.14.14

\begin{abstract}
The MYB-domain proteins exist universally across diverse organisms and regulate numerous processes during the plant life cycle. In the present research, a full-length MYB gene OsMYB511 was identified from rice seedlings through microarray data. Induction of OsMYB511 by cold stress was dramatic in japonica cultivar Jiucaiqing as compared to indica IR26. In addition to cold, OsMYB511 was also markedly induced by osmotic stress, high temperature, and exogenous ABA, suggesting that $O s M Y B 511$ is a multiple-stress responsive gene in rice. Tissue-specific expression analysis indicated that OsMYB511 was highly expressed in rice panicles at earlier development stage. Interestingly, OSMYB511 expression is fully subjected to circadian rhythm regulation. The subcellular localization and yeast hybrid assay suggested that OsMYB511 is nucleus-localized transcription activator. Deletion analysis suggested that trans-activation activity of OsMYB511 relied on its $\mathrm{C}$-terminus. Co-expression analysis revealed additional 2 MYB genes co-expressed with OsMYB511, implying that these MYB genes might coordinately regulate stress responses in rice.
\end{abstract}

Key words: Abiotic stress; Cold; MYB; Rice; Transcription factor 


\section{INTRODUCTION}

Environmental stresses including cold, drought, and high salinity are major factors affecting plant growth, development, and crop productivity. In order to overcome these adverse stress factors, plants respond and adapt to these stresses at the physiological, biochemical, and molecular levels. Stress-inducible genes may play various and important roles in response to these stresses. It is crucial to characterize the roles of these stress-regulated genes in order to develop transgenic crops with enhanced tolerance to unfavorable growth conditions. Transcription factors are crucial proteins involved in regulating gene expression at the transcriptional level by specifically binding to the regulatory promoter regions of their target genes. Several types of transcription factors, such as MYB (Yang et al., 2012), NAC (NAM ATAF1/2 CUC2) (Hu et al., 2008), WRKY (Rushton et al., 2012), zinc finger (Huang et al., 2008, 2009), and AP2/EREBP (Zhu, 2002), are known to transcriptionally regulate gene expression in response to abiotic stresses in plants.

MYB transcription factors are widely present in eukaryotes (Lippold et al., 2009). A genome-wide analysis identified at least 155 and 197 MYB genes in rice and Arabidopsis, respectively (Katiyar et al., 2012). MYB proteins contain 1, 2, or 3 imperfect repeats (51 to 53 amino acids) in their DNA-binding domain, and they are further classified into 3 subfamilies, including type MYBR2R3, type MYBR1R2R3, and MYB-related, depending on the number of repeats in their MYB domains (Stracke et al., 2001; Chen et al., 2006). A number of MYB genes were found to be regulated by abiotic stress, and their genetic engineering improved stress tolerance in plants. Rice OsMYB3R-2 is a stress-responsive MYB protein that has been well-studied. OSMYB3R-2 expression is induced by cold, drought, and salt stress. The Arabidopsis and rice transgenic plants overexpressing $O S M Y B 3 R-2$ showed increased stress tolerance (Dai et al., 2007; Ma et al., 2009). Further studies showed that OsMYB3R-2 functions as a MYB3R transcription factor targeting to $O s C y c B 1 ; 1$ (Planchais et al., 2002), which is involved in the $\mathrm{G} 2 / \mathrm{M}$ phase transition at low temperature. The transcript level of $O s C P T 1$, a putative member of the DREB1/CBF pathway, was also enhanced by OsMYB3R-2 (Ma et al., 2009). Recently, another rice MYB gene $O S M Y B 2$ was functionally examined. Expression of $O s M Y B 2$ was upregulated by salt, cold, and dehydration stress. Overexpression of OsMYB2 increased salt, cold, and dehydration tolerance. The enhanced proline and soluble sugar accumulations as well as activities of antioxidant enzymes may be responsible for the enhanced stress tolerance (Yang et al., 2012). In addition to Arabidopsis and rice, several MYB genes from other plant species, such as apple, wheat, and tobacco, may have roles in improving stress tolerance (Zhang et al., 2012; Huang et al., 2013; Wang et al., 2014).

In this study, a novel MYB gene induced by various abiotic stress, particularly dramatically induced by cold stress, was cloned from rice. Interestingly, OsMYB511 co-expressed with 2 other MYB genes. Our results indicated that OSMYB511 is an early regulator of stress responses, particularly the cold response, in rice.

\section{MATERIAL AND METHODS}

\section{Plant material and abiotic treatments}

The seeds of rice (O. sativa L. sub japonica) cultivar Jiucaiqing were sterilized in $0.1 \% \mathrm{HgCl}_{2}$ and germinated at $30^{\circ} \mathrm{C}$. The seedlings were cultured with Yoshida's culture solu- 
tion in a growth chamber with a light cycle of $16 \mathrm{~h} \mathrm{light} \mathrm{at} 30^{\circ} \mathrm{C}$ and $8 \mathrm{~h}$ dark at $22^{\circ} \mathrm{C}$. Threeleaf stage seedlings were used for stress treatments in the growth chamber with continuous light. For salt, polyethylene glycol, and abscisic acid (ABA) treatments, seedlings were transferred to nutrient solutions containing $100 \mathrm{mM} \mathrm{NaCl}, 20 \%$ (w/v) polyethylene glycol 6000 , or $50 \mu \mathrm{M}$ ABA in growth chamber at $30^{\circ} \mathrm{C}$. For cold and high temperature treatments, seedlings were transferred to the growth chamber at 4 and $42^{\circ} \mathrm{C}$, respectively.

\section{RNA isolation and first strand cDNA synthesis}

Total RNA was extract using Trizol reagent (Invitrogen, Carlsbad, CA, USA) according to the manufacturer protocol. The RNA was subsequently treated with DNase I (Promega, Madison, WI, USA) to remove the remaining genomic DNA. First-strand cDNA was synthesized with $2 \mu \mathrm{g}$ total RNA using the reverse transcription system (Promega) according to the manufacturer protocol.

\section{Cloning of OsMYB511}

The gene was cloned from cDNA prepared from rice seedlings by polymerase chain reaction (PCR) using the primers 5'-(CTCGAG)GAGATGGCGCGTTT-3' and 5'-(GTCGAC) TAAGCACAGCCTCGTCAT-3'. The PCR conditions for amplifying OsMYB511 were as follows: 5 min pre-denaturation at $98^{\circ} \mathrm{C} ; 28$ cycles for $10 \mathrm{~s}$ at $98^{\circ} \mathrm{C}, 15 \mathrm{~s}$ at $60^{\circ} \mathrm{C}, 1 \mathrm{~min} 30 \mathrm{~s}$ at $72^{\circ} \mathrm{C} ; 10$ min extension at $72^{\circ} \mathrm{C}$. The PCR product was purified and cloned into the pEASY-T vector (Transgen Biotech, Beijing, China) for sequencing.

\section{Multiple sequence alignment and phylogenetic analysis}

Multiple sequence alignment of rice and other MYB-like domain protein sequences was performed using the ClustalX program and viewed using the GeneDoc software. The molecular phylogenetic tree was constructed using the MEGA program with the neighbor-joining method. The parameters of pairwise deletion and P-distance model were used. The bootstrap test of phylogeny was performed with 1000 replicates.

\section{Real-time quantitative PCR (qPCR) analysis}

Real-time qPCR was carried out in a total volume of $20 \mu \mathrm{L}$ containing $1 \mu \mathrm{L}$ cDNA, $0.4 \mu \mathrm{M}$ gene-specific primers, $10 \mu \mathrm{L}$ SYBR Green Real-Time PCR Master Mix, and $2 \mu \mathrm{L}$ plus solution (Toyobo Co., Ltd., Osaka, Japan) using an ABI7500 Fast Real-time PCR System (Applied Biosystems, Foster City, CA, USA). The rice 18S rRNA gene was used as the internal control. The primers for OsMYB511 were 5'-AAGCGTAAGCCTGTTCATC-3' and 5'-CCAGTGTATCAGCCCGTAT-3'. Relative quantification of the transcript level was determined using the comparative $\mathrm{Ct}$ method.

\section{Subcellular localization}

The green fluorescent protein (GFP) fusion protein was constructed using the fulllength OsMYB511 cDNA clone with a C-terminal fusion of the GFP clone under control of the 
cauliflower mosaic virus $35 \mathrm{~S}$ promoter. The primers used for OsMYB511 subcellular localization analysis were 5'-AAGCGTAAGCCTGTTCATC-3' and 5'-CCAGTGTATCAGCCCGTA T-3'. The construct was confirmed by sequencing and used for transient transformation of onion epidermis using a gene gun (Bio-Rad, Hercules, CA, USA). After $24 \mathrm{~h}$ of incubation, GFP fluorescence in transformed onion cells was observed under a fluorescence microscope (ECLIPSE 80i; Nikon, Tokyo, Japan).

\section{Transcriptional activation assay}

DNA fragments containing the whole or partial open reading frame of OsMYB511 were cloned into the pGBKT7 vector to obtain the pGBKT7-OsMYB511, pGBKT7OsMYB511-N (1 to 106 amino acids), and pGBKT7-OsMYB511-C (107 to 464 amino acids) constructs. Using the lithium acetate-based method, all constructs, including pGBKT7OsMYB511, pGBKT7-OsMYB511-N, pGBKT7-OsMYB511-C, the positive control pGADT7-T+pGBKT7-53, and the negative control pGBKT7 were transferred into the yeast strain AH109. Transformed yeast cells were grown on SD/-Trp, SD/-Trp-His-Ade plates. The trans-activation activity of each protein was evaluated according to its growth status and $\alpha$-galactosidase activity as indicated by the chromogenic substrate $\mathrm{X}$ - $\alpha$-gal in the $\mathrm{SD} /-$ Trp-His-Ade plates.

\section{Co-expression analysis}

Co-expression analysis was performed using the Rice Oligo Array Database (http://ricearray.org). The genes co-expressed with OSMYB511 were selected using a Pearson's correlation coefficient cutoff of 0.6 . The expression changes of co-expressed genes under cold, salt, and drought stresses were investigated based on our microarray data (unpublished).

\section{RESULTS}

\section{Isolation and sequence analysis of OsMYB511}

Using microarray data of rice seedling under cold stress (our unpublished data), we identified an expressed sequence tag probe (ID: Os.5335.1.S1_at) dramatically induced by cold stress ( $\sim 121$ folds). Database searching indicated that this expressed sequence tag probe corresponds to an MYB-domain protein. Sequence analysis indicated that this gene belongs to the R1-type subfamily of MYB proteins. OsMYB511 consists of a 1389-base pair coding region encoding a peptide of 463 amino acids. The molecular mass calculated of the OsMYB511 protein was found to be $51.1 \mathrm{kDa}$.

Multiple sequence alignment showed that OsMYB511 is homologous to numerous MYB transcription factors containing single repeats in the domain (Figure 1). A phylogenetic tree was constructed using the neighbor-joining method with the full-length amino acid sequences of some well-studied MYB proteins (Figure 2), which revealed that OsMYB511 was clustered closely to AtRVE1 (Rawat et al., 2009), GmMYB177 (Liao et al., 2008), and ZmCCA1 (Wang et al., 2011). 


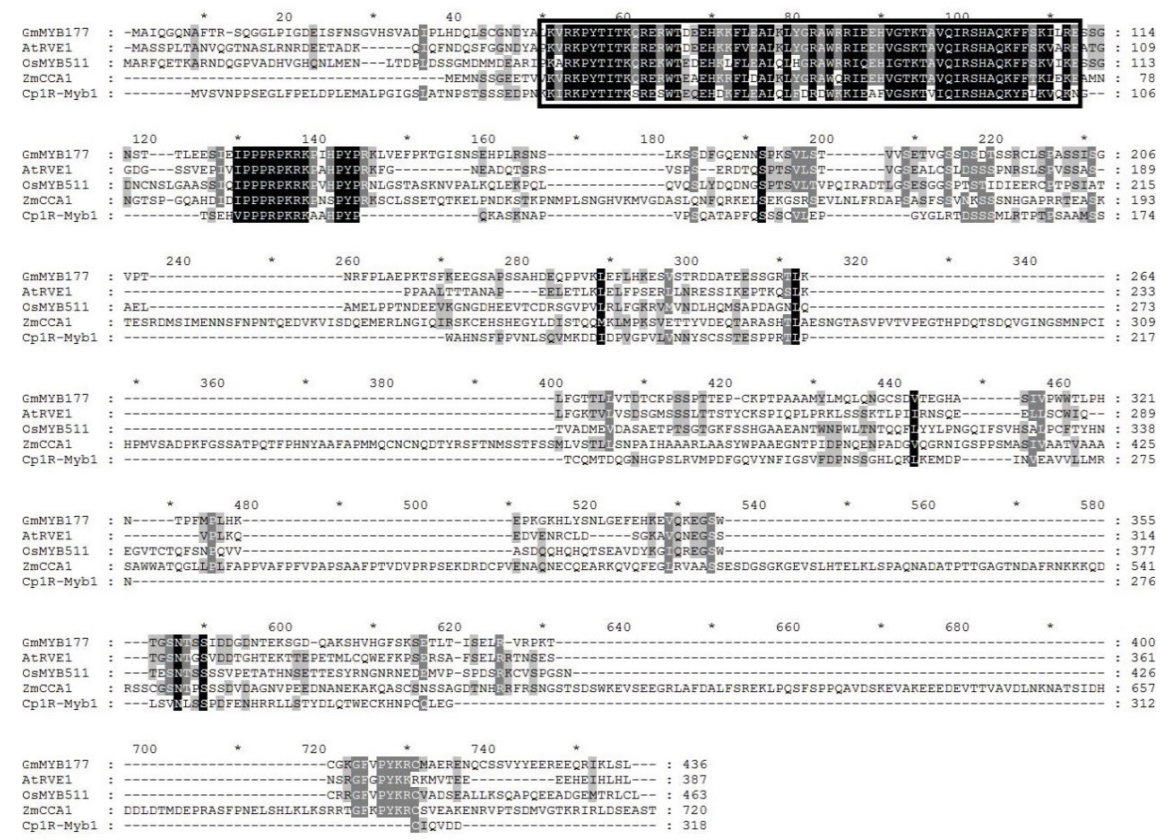

Figure 1. Sequence alignment of OsMYB511 and other plant MYB-domain-containing proteins. The alignment was constructed using the ClustalX program with amino acid sequences. The SANT domain is boxed.

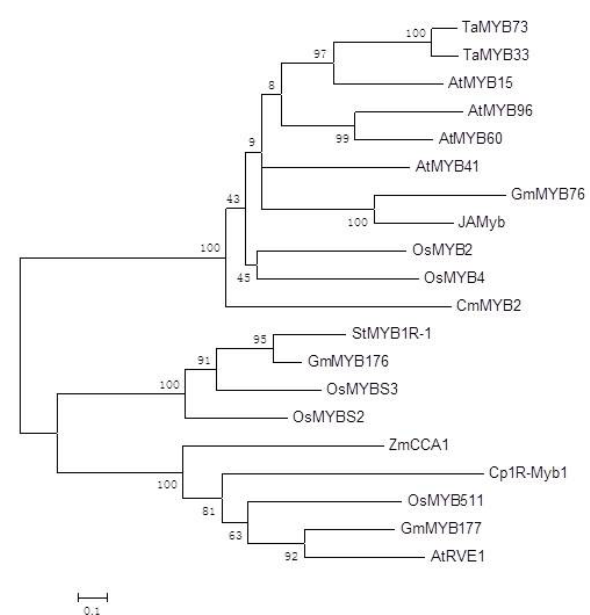

Figure 2. Phylogenetic analysis of MYB-domain-containing proteins. The tree was constructed using the MEGA program with amino acid sequences of plant stress-related MYB proteins. Branch numbers represent a percentage of the bootstrap values in 1000 sampling replicates, and the scale bar indicates the branch length. GenBank accession No. for the protein sequences: Oryza sativa - OsMYBS3 (AAN63154.1), OsMYB4 (BAA23340.1), OsMYB2 (BAA23338.1), JAMyb (AAK08983.1), and OsMYBS2 (AAN63153.1); Triticum aestivum - TaMYB73 (AEW23186.1) and TaMYB33 (AEV91156.1); Glycine max - GmMYB177 (ABH02866.1), GmMYB76 (ABH02836.1), and GmMYB176 (NP_001236048.1); Arabidopsis thaliana - AtRVE1 (AED92410.1), AtMYB96 (AED97611.1),AtMYB41 (EFH43724.1),AtMYB15 (AEE76741.1), and AtMYB60 (AEE28351.1); Zea mays ZmCCA1 (ADU60099.1); Chimonanthus praecox - Cp1R-Myb1 (ABV02070.1); Chrysanthemum x morifolium - CmMYB2 (AEO27498.1); and Solanum tuberosum - StMYB1R-1 (Q2V9B0.1). 


\section{OsMYB511 is highly expressed in the panicles at early developmental stage}

To determine the expression pattern of OSMYB511 in rice, we analyzed the expression levels of OSMYB511 in various tissues of rice using real-time PCR. The results showed that OsMYB511 was expressed in all tissues examined, including the roots, stems, leaves, leaf sheathes, and panicles (Figure 3). Interestingly, OSMYB511 accumulated to higher levels in rice panicles less than $3 \mathrm{~cm}$, but was lowly expressed in the panicles that were more than $3 \mathrm{~cm}$ (Figure 3), suggesting that OsMYB511 functions specifically in the panicles during the early development stage.

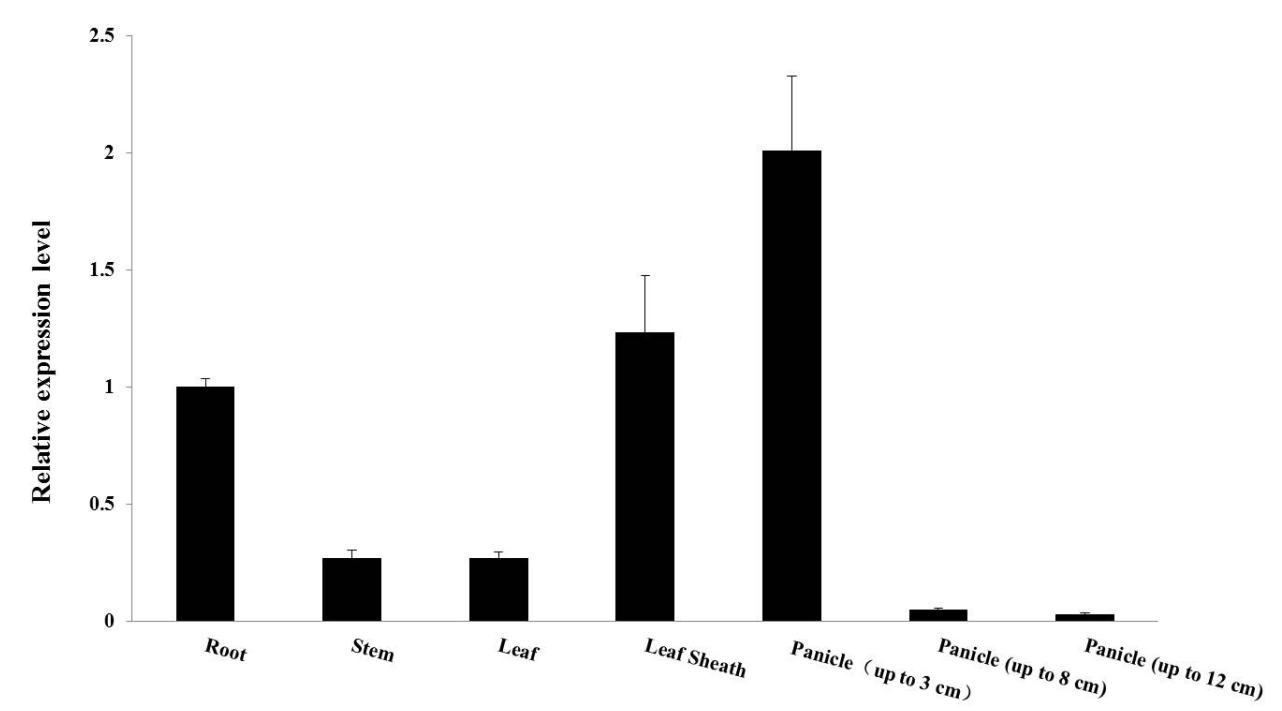

Figure 3. qRT-PCR analysis of tissue-specific expression of OsMYB511.

\section{Expression of OsMYB511 in rice under cold stress}

To confirm the stress-responsive expression pattern observed in microarray analysis, we analyzed the expression levels of OsMYB511 in 2 rice cultivars under cold stress. In the japonica cultivar Jiucaiqing, OSMYB511 showed a very quick and dramatic induction after being subjected to cold treatment (Figure 4). Cold stress treatment for 20 min induced OSMYB511 expression by approximately 40-fold and OSMYB511 transcripts accumulated to the maximum levels after treatment for $3 \mathrm{~h}$, when induction reached approximately 140 -fold. In the indica cultivar IR26, OsMYB511 was induced much more slowly and only slightly by cold stress (Figure 4). This result suggested that OsMYB511 plays a role on a different scale in response to cold stress in 2 rice subspecies.

\section{Expression of $O S M Y B 511$ in rice under other abiotic stress}

In addition to cold stress, OSMYB511 was also significantly induced after treatment with polyethylene glycol 6000, high temperature, and ABA treatment (Figure 5). The tran- 
scripts levels of OsMYB511 were increased within $20 \mathrm{~min}$ and peaked at 1 or $3 \mathrm{~h}$, and then decreased. For exogenous ABA treatment, the transcript level of OsMYB511 peaked within 15 min and then decreased. For salt stress, expression of OSMYB511 was slightly induced after 3 $\mathrm{h}$ of treatment and decreased thereafter. Our data suggest that OsMYB511 probably plays an important role during the early stage of various stress responses in rice.

Jiucaiqing

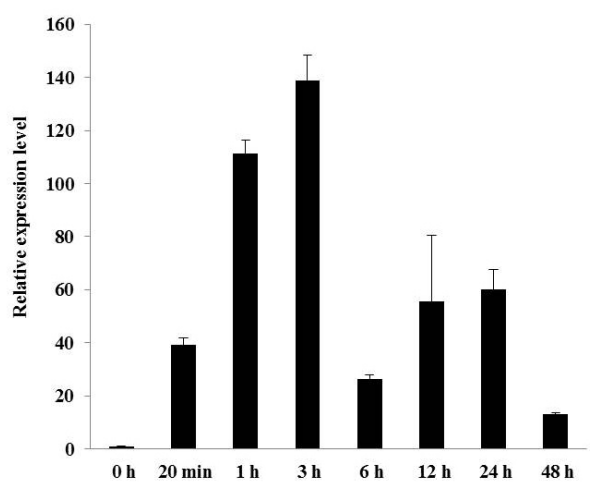

IR-26

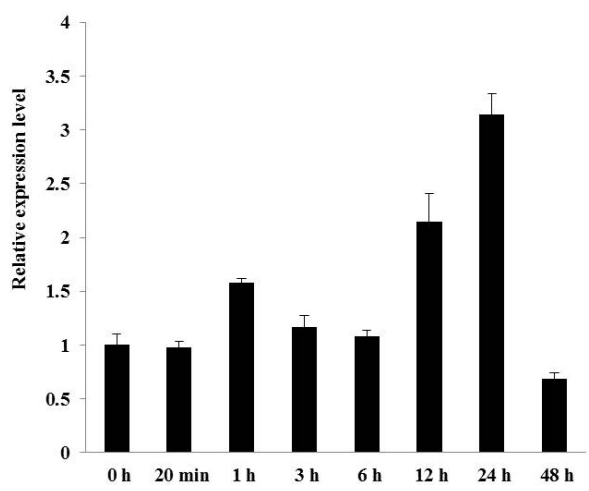

Figure 4. Comparative expression analysis of OSMYB511 gene in 2 rice cultivars under cold stress.
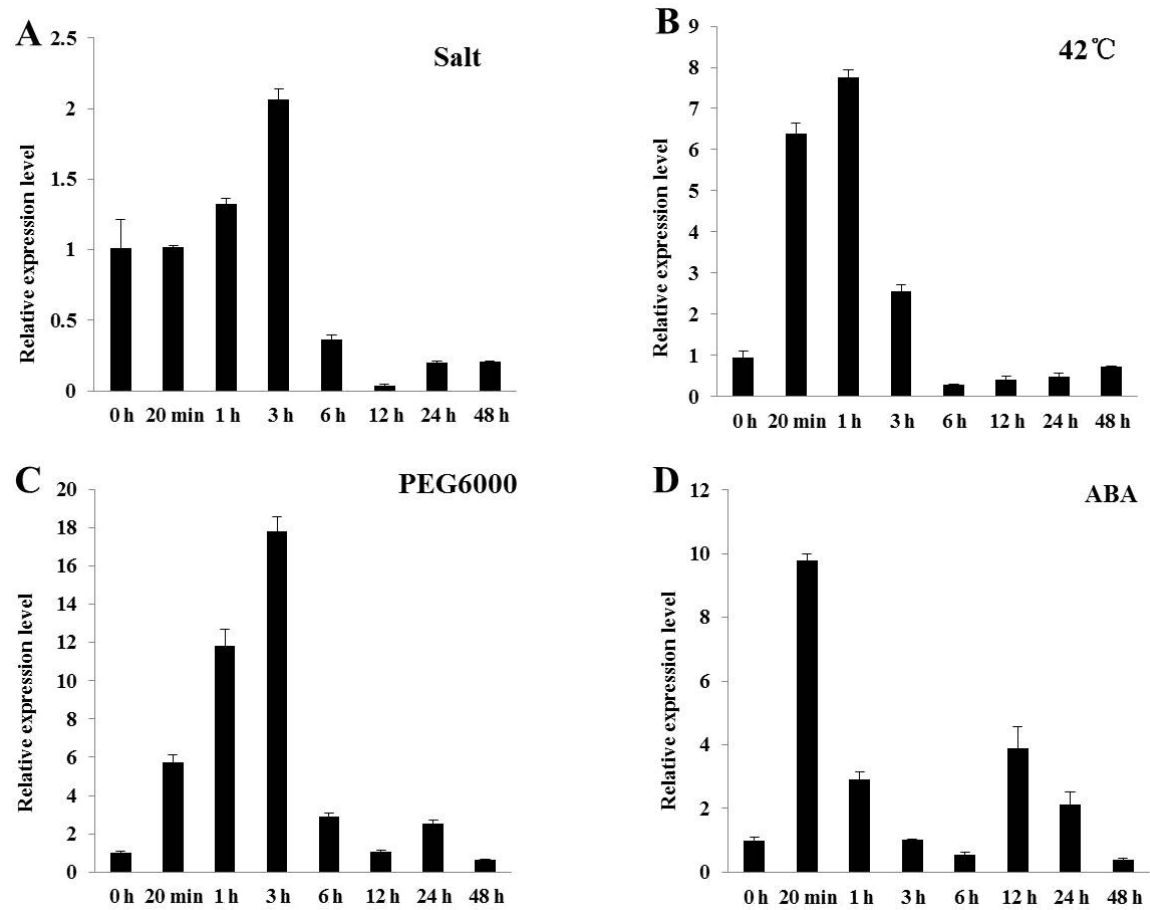

Figure 5. Expression analysis of OSMYB511 gene in rice under abiotic stresses. qRT-PCR was performed with rice (japonica Jiucaiqing) seedlings treated with salt (A), high temperature (B), polyethylene glycol 6000 (C), and ABA(D). 


\section{Expression of $O S M Y B 511$ is involved in circadian rhythm}

Analysis using RiceXPro (Sato et al., 2013) showed that expression of OsMYB511 was significantly regulated by the circadian rhythm. The OSMYB511 gene was highly expressed in the middle of the night ( midnight) and lowly expressed during the middle of the day ( midday) (Figure 6$)$.

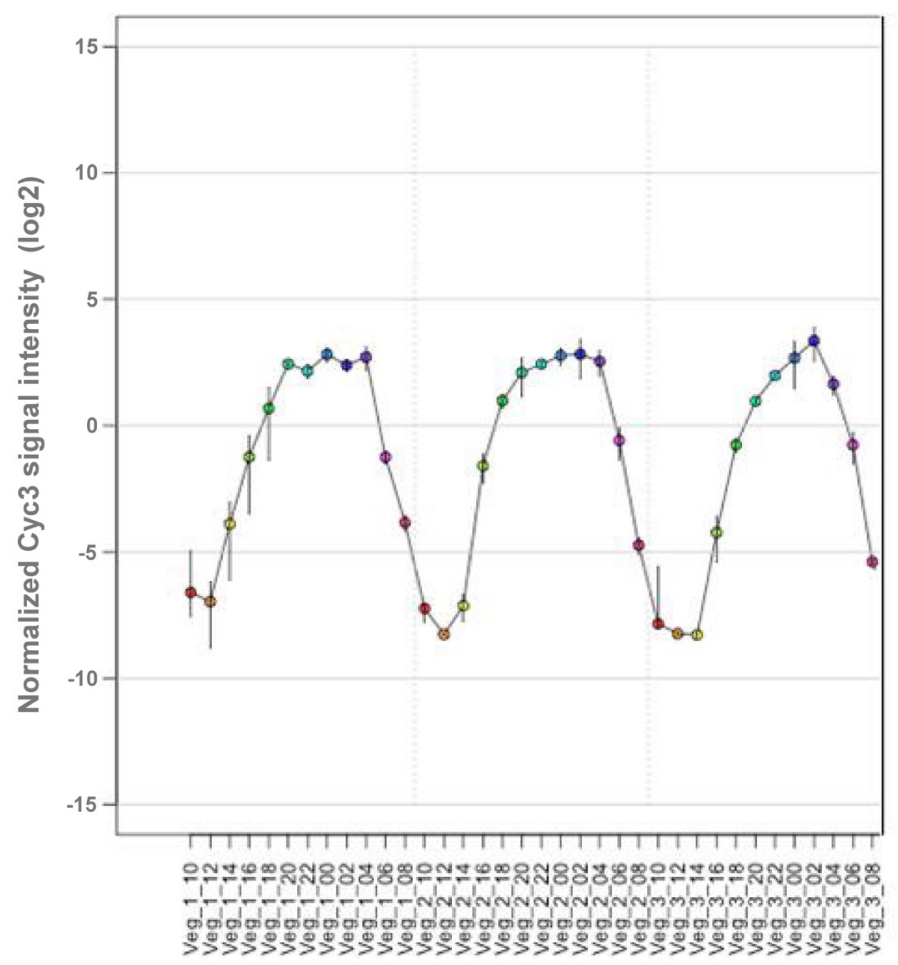

Figure 6. Expression of OsMYB511 in rice response to circadian rhythm in rice leaves. The gene expression analysis was based on RiceXPro microarray data (Sato et al., 2013).

\section{OsMYB511 is located in the nucleus}

Because OsMYB511 encodes a putative MYB transcription factor, we analyzed the subcellular localization of OsMYB511. The full-length OSMYB511 was in-frame and fused with the GFP reporter gene and then transiently expressed in onion epidermal cells. As shown in Figure 7, the OsMYB511-GFP fusion protein was specifically localized to the nucleus, whereas GFP alone was distributed throughout the whole cell.

\section{OsMYB511 is a transcription activator}

To determine whether OsMYB511 functions as a transcription factor, we applied the yeast 2-hybrid system to investigate the trans-activation potential of OsMYB511. A GAL4 
DNA binding domain-OsMYB511-fused protein, was expressed in AH109 yeast cells, and then grown on $\mathrm{SD} /$-Trp and $\mathrm{SD} /$-Trp-His-Ade medium to test whether the fused protein could activate transcription of the reporter gene. As shown in Figure 8, the full-length OsMYB511 (pGBKT7-OsMYB511) grew on SD/Trp-/His-/Ade medium and showed $\alpha$-galactosidase activity, confirming that OsMYB511 functions as a transcriptional activator in yeast cells.

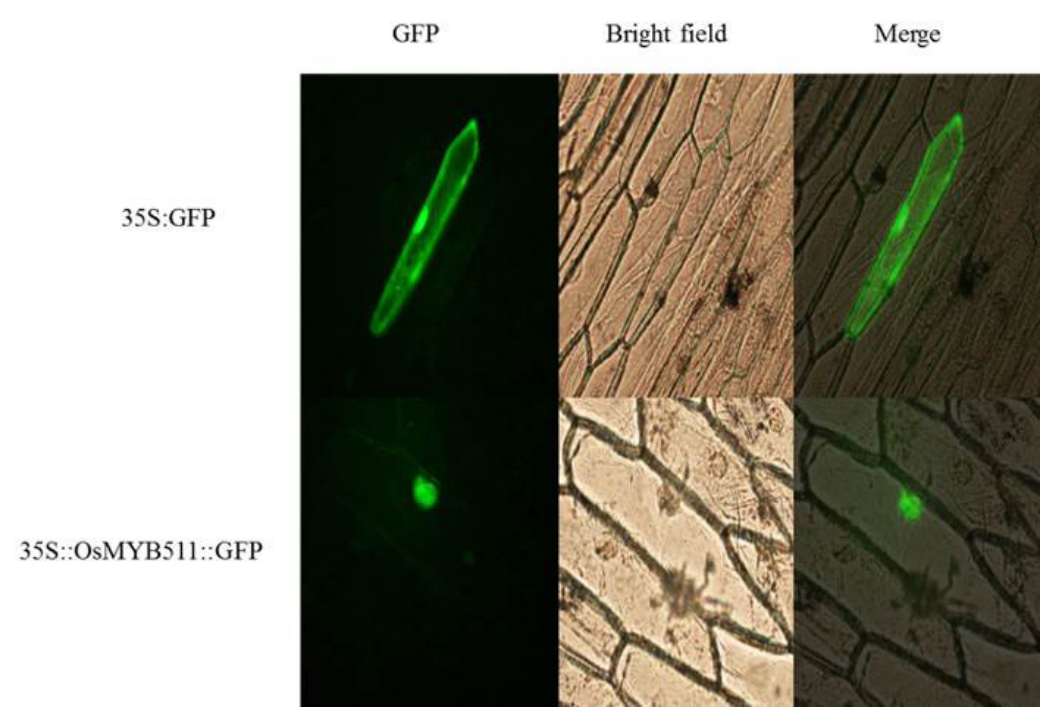

Figure 7. Nuclear localization of GFP-OsMYB511 fusion protein.

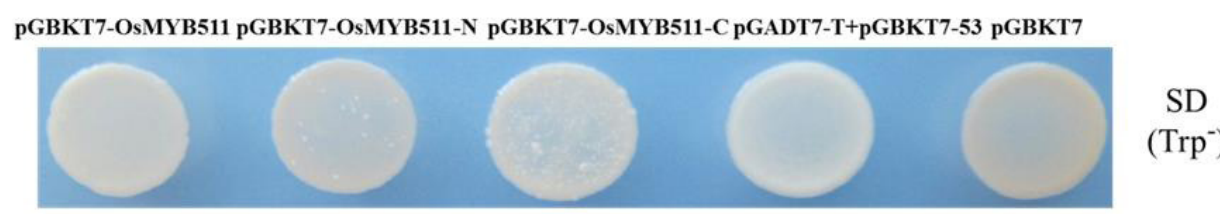

pGBKT7-OsMYB511 pGBKT7-OsMYB511-N pGBKT7-OsMYB511-C pGADT7-T+pGBKT7-53 pGBKT7

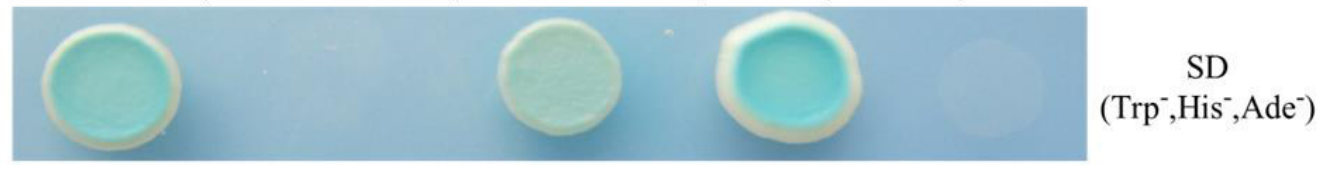

Figure 8. OsMYB511 showed transcription activation activity and the C-terminus was required for trans-activation.

\section{C-terminus of OsMYB511 functions as activation region}

To determine whether transcriptional activity was related to the MYB domain of OsMYB511, OsMYB511 lacking the MYB domain OsMYB511-C (107 to 464), and OsMYB511 lacking the C-terminal region OsMYB511-N (1 to 106) were used to evaluate transcriptional activity. The OsMYB511 lacking the MYB domain OsMYB511-C (107 to 464) 
grew on $\mathrm{SD} / \mathrm{Trp}$-/His-/Ade medium and exhibited $\alpha$-galactosidase activity, confirming that the activation domain of OsMYB511 is related to the C-terminal region.

\section{Two MYB genes were co-expressed with OsMYB511}

Based on co-expression analysis using the Rice Oligonucleotide Array Database, a total of 16 genes were found to be co-expressed with OSMYB511 with Pearson's correlation coefficient higher than 0.6 (data not shown). Interestingly, we found that 2 other MYB genes (LOC_Os08g04840, LOC_Os02g46030) were co-expressed with OsMYB511 with higher Pearson's correlation coefficients $(>0.75)$. OsMYB511 showed higher sequence similarity to LOC_Os02g46030, but low sequence homology with LOC_Os08g04840. However, these MYB genes were significantly upregulated by cold stress (Figure 9).
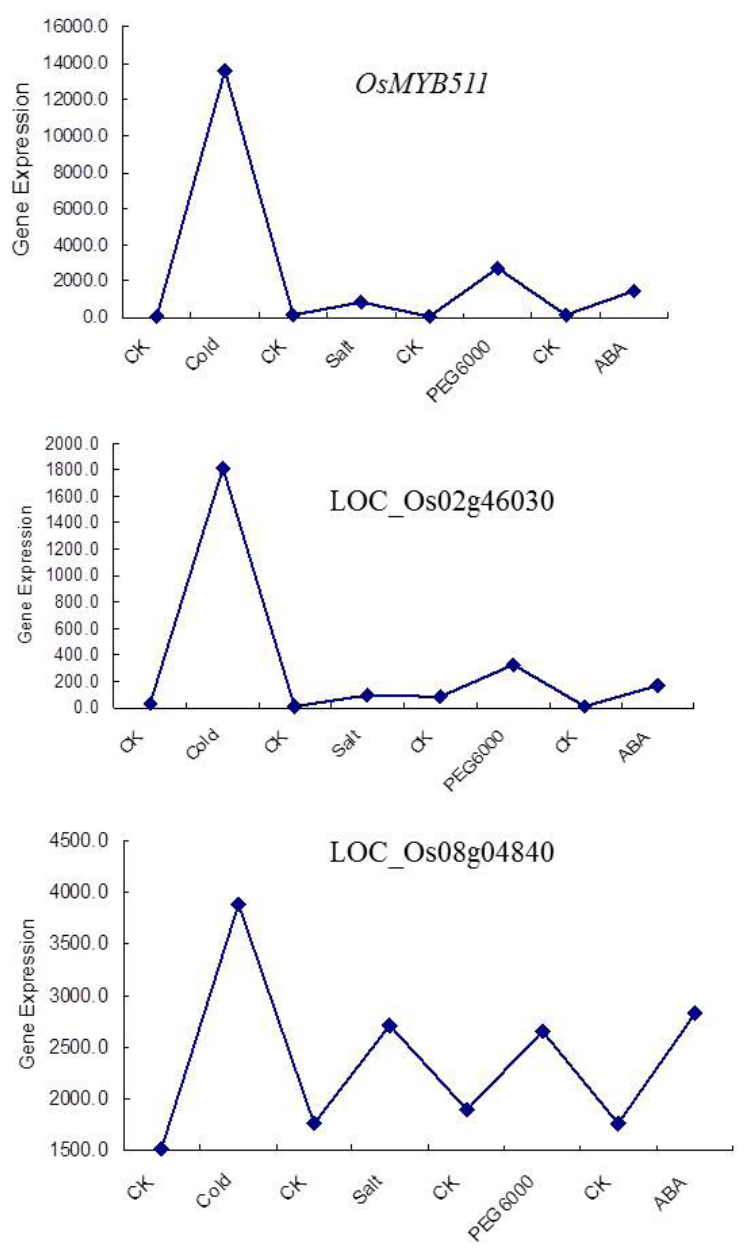

Figure 9. Expression of 3 co-expressed MYB genes in rice under abiotic stress. The expression data is based on our microarray analysis of rice (japonica rice Zhonghua 11) seedlings subjected to abiotic stresses $\left(4^{\circ} \mathrm{C}\right.$ for cold stress; $150 \mathrm{mM} \mathrm{NaCl}$ for salt stress, $20 \%$ polyethylene glycol 6000 for osmotic stress, and $0.1 \mathrm{mM} \mathrm{ABA}$ for ABA treatment). 


\section{DISCUSSION}

In the present study, we identified a new MYB protein involved in the stress response, particularly the cold response in rice. Using multiple sequence alignment and phylogenetic analysis, we found that OsMYB511 is most closely related to AtRVE1 (Rawat et al., 2009), GmMYB177 (Liao et al., 2008), and ZmCCA1 (Wang et al., 2011). Similar to OsMYB511, AtRVE1 is involved in the circadian rhythm. These results suggest that this MYB subfamily may specifically involve the circadian rhythm in plants. The subcellular localization and yeast hybrid assay results suggested that OsMYB511 is a nucleus-localized transcription activator. Deletion analysis indicated that the C-terminus without the MYB domain conferred transactivation activity, suggesting that the MYB domain is not necessary for transcriptional activation, while the $\mathrm{C}$-terminal region is probably responsible for transcriptional activation activity.

Stress responsive expression of OSMYB511 indicated that OSMYB511 likely functions in response to environmental stimuli. The OsMYB511 gene was induced very rapidly by abiotic stresses and exogenous ABA treatment, suggesting that it may function at the upstream level of the signal transduction pathway in rice under abiotic stress. OsMYB511 was transiently induced by stresses and its expression recovered to baseline levels within $6 \mathrm{~h}$. Hence, OsMYB511 may rapidly sense and transduce the stress signal in rice and overproduction of OsMYB511 may be not necessary for stress tolerance or may play a negative role in plant growth. Compared with other environmental stresses, OsMYB511 was dramatically induced by cold stress in the rice cultivar Jiucaiqing, implying that the function of OsMYB511 may be more specific under cold stress. However, in the indica cultivar IR26, OsMYB511 was slightly induced by cold. The different induction levels in 2 rice cultivars indicate that OsMYB511 plays roles to different extents in cold tolerance. Interestingly, OsMYB511 was significantly regulated by circadian rhythm. As OsMYB511 is largely induced upon cold stress, we hypothesized that OsMYB511 promotes cold-responsive gene expression to be resistant to low temperature at night. With temperature increases during the day, expression of OsMYB511 gradually decreases to avoid the negative effect of OsMYB511 in plant growth and development. Interestingly, Arabidopsis orthologs of OsMYB511 and REVEILLE1 (RVE1) (Rawat et al., 2009), are also involved in circadian rhythm. These data imply a general mechanism for MYB proteins in response to cold stress, which, however, requires further investigation. Through expression analysis, our data also showed that OSMYB511 was induced by high temperature to a much lower extent than with cold induction. Expression of OsMYB511 may have been upregulated when temperature was over-high, resulting in heat stress. In addition to cold and heat stress, OsMYB511 was induced by other major environmental stresses, including salt, drought, and hormone ABA treatment. These treatments typically cause osmotic stress to plant cells, and induction by ABA indicated that OsMYB511 functions in an ABA-dependent manner.

Co-expression analysis suggested that a number of genes involved in abiotic stress were co-expressed with OSMYB511. Interestingly, 2 additional MYB genes co-expressed with OsMYB511. These genes were all significantly induced by cold stress, implying they have coordinate and predominant roles in cold tolerance in rice. Whether the 3 MYB genes are functionally redundant requires further analysis using single-, double-, and/or triple-myb mutants.

In summary, a novel MYB gene OsMYB511 was identified and characterized in the present study. OsMYB511 is a nucleus-localized transcription activator. Expression analysis suggested that OSMYB511 was highly expressed in the panicles during the early developmental stage and dramatically induced by cold stress, particularly in japonica rice. Two additional 
MYB genes were found to be co-expressed with OsMYB511 and were both markedly regulated by cold stress. Our data suggest that OsMYB511 plays important roles in regulating stress-responsive gene expression and panicle development in rice. Further transgenic studies are necessary to elucidate the biological function of OSMYB511 and its potential value in engineering rice varieties with enhanced stress tolerance.

\section{ACKNOWLEDGMENTS}

Research supported by the Jiangsu Collaborative Innovation Center for Modern Crop Production, National Science Foundation of China (\#31071069), Jiangsu Provincial Natural Science Foundation (\#BK20141362), and the Fundamental Research Funds for the Central Universities (\#KYZ201137).

\section{REFERENCES}

Chen J, Kremer CS and Bender TP (2006). The carbonic anhydrase I locus contains a c-Myb target promoter and modulates differentiation of murine erythroleukemia cells. Oncogene 25: 2758-2772.

Dai X, Xu Y, Ma Q, Xu W, et al. (2007). Overexpression of an R1R2R3 MYB gene, OsMYB3R-2, increases tolerance to freezing, drought, and salt stress in transgenic Arabidopsis. Plant Physiol. 143: 1739-1751.

Hu H, You J, Fang Y, Zhu X, et al. (2008). Characterization of transcription factor gene SNAC2 conferring cold and salt tolerance in rice. Plant Mol. Biol. 67: 169-181.

Huang J, Wang MM, Jiang Y, Bao YM, et al. (2008). Expression analysis of rice A20/AN1-type zinc finger genes and characterization of ZFP177 that contributes to temperature stress tolerance. Gene 420: 135-144.

Huang J, Sun SJ, Xu DQ, Yang X, et al. (2009). Increased tolerance of rice to cold, drought and oxidative stresses mediated by the overexpression of a gene that encodes the zinc finger protein ZFP245. Biochem. Biophys. Res. Commun. 389: 556-561.

Huang W, Sun W, Lv H, Luo M, et al. (2013). A R2R3-MYB transcription factor from Epimedium sagittatum regulates the flavonoid biosynthetic pathway. PLoS One 8: e70778.

Katiyar A, Smita S, Lenka SK, Rajwanshi R, et al. (2012). Genome-wide classification and expression analysis of MYB transcription factor families in rice and Arabidopsis. BMC Genomics 13: 544.

Liao Y, Zou HF, Wang HW, Zhang WK, et al. (2008). Soybean GmMYB76, GmMYB92, and GmMYB177 genes confer stress tolerance in transgenic Arabidopsis plants. Cell Res. 18: 1047-1060.

Lippold F, Sanchez DH, Musialak M, Schlereth A, et al. (2009). AtMyb41 regulates transcriptional and metabolic responses to osmotic stress in Arabidopsis. Plant Physiol. 149: 1761-1772.

Ma Q, Dai X, Xu Y, Guo J, et al. (2009). Enhanced tolerance to chilling stress in OsMYB3R-2 transgenic rice is mediated by alteration in cell cycle and ectopic expression of stress genes. Plant Physiol. 150: 244-256.

Planchais S, Perennes C, Glab N, Mironov V, et al. (2002). Characterization of cis-acting element involved in cell cycle phase-independent activation of Arath; $\mathrm{CycB} 1 ; 1$ transcription and identification of putative regulatory proteins. Plant Mol. Biol. 50: 109-125.

Rawat R, Schwartz J, Jones MA, Sairanen I, et al. (2009). REVEILLE1, a Myb-like transcription factor, integrates the circadian clock and auxin pathways. Proc. Natl. Acad. Sci. U. S. A. 106: 16883-16888.

Rushton DL, Tripathi P, Rabara RC, Lin J, et al. (2012). WRKY transcription factors: key components in abscisic acid signalling. Plant Biotechnol. J. 10: 2-11.

Sato Y, Takehisa H, Kamatsuki K, Minami H, et al. (2013). RiceXPro version 3.0: expanding the informatics resource for rice transcriptome. Nucleic Acids Res. 41: D1206-1213.

Stracke R, Werber M and Weisshaar B (2001). The R2R3-MYB gene family in Arabidopsis thaliana. Curr. Opin. Plant Biol. 4: 447-456.

Wang RK, Cao ZH and Hao YJ (2014). Overexpression of a R2R3 MYB gene MdSIMYB1 increases tolerance to multiple stresses in transgenic tobacco and apples. Physiol. Plant 150: 76-87.

Wang X, Wu L, Zhang S, Wu L, et al. (2011). Robust expression and association of ZmCCA1 with circadian rhythms in maize. Plant Cell Rep. 30: 1261-1272.

Yang A, Dai X and Zhang WH (2012). A R2R3-type MYB gene, OsMYB2, is involved in salt, cold, and dehydration tolerance in rice. J. Exp. Bot. 63: 2541-2556. 